EGG-EE--8256

DE91 001932

\title{
DESIGN OF A NEURAL-NETWORK CONTROL SYSTEM
}

\author{
Dean C. Mumme ${ }^{a}$
}

Donald R. Chick

\section{Published September 1988}

\section{DISCLAIMER}

This report was prepared as an account of work sponsored by an agency of the United States Government. Neither the United States Government nor any agency thereof, nor any of their employees, makes any warranty, express or implied, or assumes any legal liability or responsibility for the accuracy, completeness, or usefulness of any information, apparatus, product, or process disclosed, or represents that its use would not infringe privately owned rights. Reference herein to any specific commercial product, process, or service by trade narne, trademark, manufacturer, or otherwise does not necessarily constitute or imply its endorsement, recommendation, or favoring by the United States Government or any agency thereof. The views and opinions of authors expressed herein do not necessarily state or reflect those of the United States Government or any agency thereof.

\section{MESTER rot}

a. University of Idaho 


\section{ABSTRACT}

Neural networks can be used for controllers for feedback control systems. Usually the systems being controlled are not described mathematically but some description of the system is needed in order for a neural network controller to be designed. An approach in which another neural network is used to emulate the controlled system is developed. After the neural network emulator has been developed the neural network controller is designed using this emulator. Both neural networks are designed using the backpropagation technique. 


\section{CONTENTS}

ABSTRACT

1. INTRODUCTION $\ldots \ldots \ldots \ldots \ldots \ldots \ldots \ldots \ldots \ldots \ldots \ldots \ldots \ldots \ldots \ldots \ldots \ldots$

2. SYSTEM OVERVIEW $\ldots \ldots \ldots \ldots \ldots \ldots \ldots \ldots \ldots \ldots \ldots \ldots \ldots \ldots \ldots \ldots \ldots \ldots \ldots, 2$

3. THE DYNAMIC SYSTEM: COUPLED TANKS $\ldots \ldots \ldots \ldots \ldots \ldots \ldots \ldots \ldots$

4. COURSE ARCHITECTURE OF THE EMULATOR AND CONTROLLER $\ldots \ldots \ldots \ldots \ldots$. 11

5. CONTROL SYSTEM OPERATION $\ldots \ldots \ldots \ldots \ldots \ldots \ldots \ldots \ldots \ldots \ldots \ldots \ldots \ldots \ldots \ldots \ldots \ldots$

6. FINE ARCHITECTURE OF THE EMULATOR AND CONTROLLER $\ldots \ldots \ldots \ldots \ldots, 23$

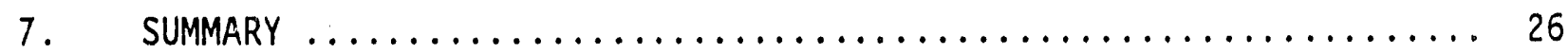

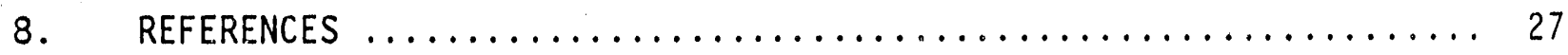

APPENDIX A--TWO TANK SYSTEM DYNAMICS $\ldots \ldots \ldots \ldots \ldots \ldots \ldots \ldots \ldots \ldots \ldots$ Al

\section{FIGURES}

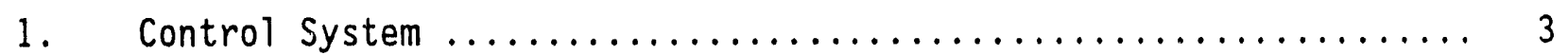

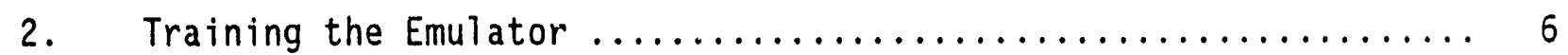

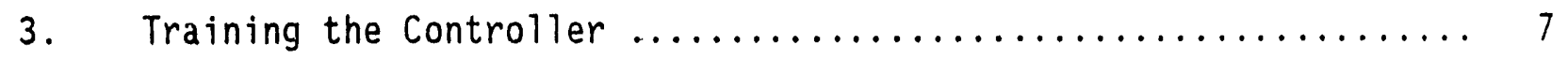

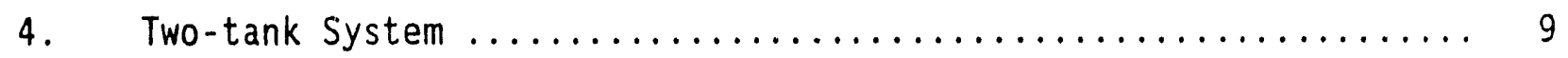


Contents (Cont'd)

5. Groups of Cells in the Emulator and Controller .............. 13

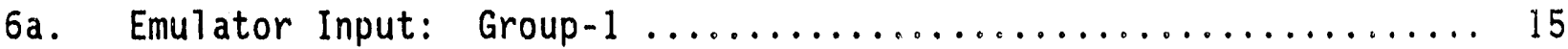

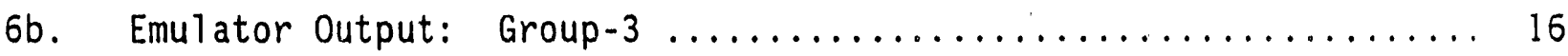

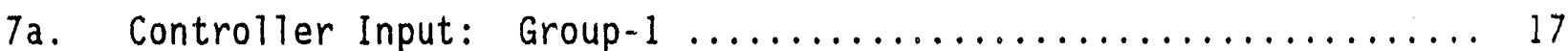

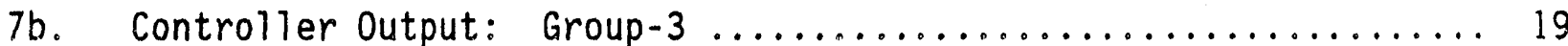

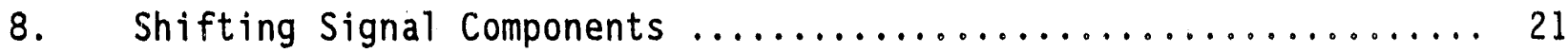

9. Structure of Signal Components $\ldots \ldots \ldots \ldots \ldots \ldots \ldots \ldots \ldots \ldots, 24$ 


\section{INTRODUCTION}

This project entailed the design and implementation of neural networks whose function is to control dynamic industrial processes. The capacity of neural networks for adaptive performance and their inherent parallel operation offers promise for the development of truly general purpose industrial control systems operating in real time. The capability of adaptive performance would allow an industrial assembler (e.g. an arc weider) to change its operational protocol after it has been programmed. This dynamic adjustment of the control system would allow the system to account for factors that are unknown at the time that the system is first brought into service. The system could thereby optimize its perfiormance for a specific task or adapt to a task for which it was not specifically designed. Parallel operation on the other hand would allow a large data flow rate from sensor inputs and allow quick control response to those inputs. This feature is necessary for controlling high speed processes (e.g. aircraft guidance) in real time.

The goals for this project were to develop a pilot neural network control system (hereafter called the "neural control system" or "neural controller") and determine useful methods of designing/training the neural controller. The dynamic system chosen for study was a system of interconnected tanks of liquid solutions. Each tank has some concentration of solute which can be monitored by the controller and an inlet valve that can be adjusted by the controller to achieve a desired concentration. The concentrations in the system of tanks constitute the state of the system and obey known dynamical equations. Use of these equations allows computer simulation of both the system of tanks and the controller on a computer to assess performance and test designs and control training strategies. 
The strength of tnis approach is that the controller is not assumed to have knowledge of the the dynamical equations of the dynamic system it is to control. The controller is essentially self programming and "learns" the correct control protocol during the control process ("learning while doing"). During this process, the dynamic system equations can be changed slightly from one simulation to the next as the controller learns in order to force the controller to adapt to new situations. As a result, errors made modelling a particular dynamic system should not adversely effect the controller's performance when it is put to use on a "real world" system.

The tank system was chosen for its simplicity with the intent of controlling more complex systems as the design techniques are identified and perfected. However, control of the tank system is important in itself for industrial control of continuous processes. These are processes in which parameters such as concentrations, temperatures, pressures, etc. must be regulated over time to manufacture products such as plastics and other chemical products, metal alloys, bacteria cultures, pharmaceutical products, and silicon microchips to name a few. Non-manufacturing industrial applications include power plant operation (nuclear or otherwise) and the control of auto emissions. With this in mind, the proposed methods for adaptive process control are intended to be general purpose. Techniques developed for one application will be adaptable to a wide range of others in a straight forward manner.

\section{SYSTEM OVERVIEW}

We will begin by describing the system in general terms and then we'11 treat each component of the system in detail. Figure 1 depicts the system's most essential fcatures. The dynamic system is to perform an action determined by the user. This is indicated by the desired response signal originating from the user shown in the figure. This signal arrives at the neural controller and the comparator. The nfural controller is a neural net whose job is to send the proper control signals to the dynamic system to get the latter to perform the desired response. In the figure, this means that 


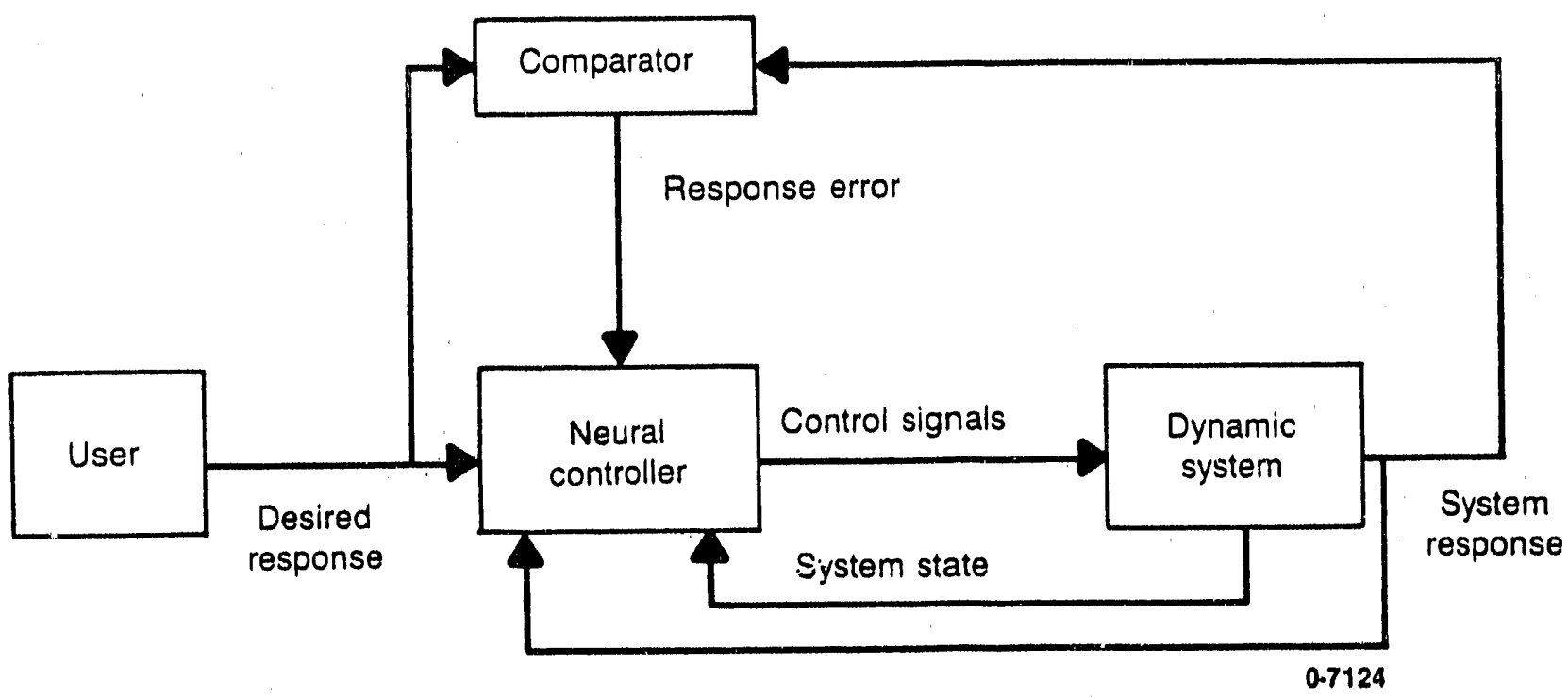

Figure 1. Control System. 
the system response must match the desired response. The comparator subtracts the system response from the desired response to obtain the error signal called the response error to the neural controller. This indicates how far away the system response is from the desired response. To compensate for the response error, the neural controiler will correct the control signals it sends to the dynamic system. The process will repeat until the response error is zero so that the system response matches the desired response.

In order to properly control the dynamic system, the neural controller must have information pertaining to the internal state of the dynamic system. This is indicated by the signal system state that runs from the dynamic system to the neural controller. Finally, the controller is also fed the system response signal in order to mnnitor the results of its efforts to control the dynamic system. In fact, the role of the comparator can be incorporated into the neural controller since both receive the system response and desired response signals. The comparator is shown separately here only to aid understanding of the system design.

The system just described is for the case that the neural controller is already capable of carrying out the control function. In this case, this system is very similar to standard control systems. However, the most important feature of the neural controller is its adaptivity which makes it different from standard control systems. The neural controller can learn to properly control the dynamic system without previously having been prograrnmed or "told" how to do it. This is much like "on the job training" where humans "learn by doing" a particular job. A neural controller when first used would begin by making errors when trying to control the dynamic system. By monitoring the errors it makes, the controller would adjust its internal parameters, or weights as they are called, untii it finds the correct control actions to do the job. Its performance wouid gradualiy improve until its control capability was fine tuned. At this point, the learning ability could be turned off and the neural controller would act as described in the preceding paragraphs. Alternatively, the controller could 
be left in the learn mode in order to learn new control tasks as operating conditions change or the user's needs change.

In order for the neural controller (or controller) to learn, the response error must be converted into a control error. This is necessary if the controller is to determine what's wrong with the control signals it is sending to the dynamic system. Figure 2 shows the control system with the addition of a neural network called the neural emulator or emulator and a comparator that determines the emiulator error. These are the components neeled to convert the response error into a control error. Before this conversion can be made, the neural emulator must first learn to imitate or emulate the dynamic system. It does so by sampling the control signals going to the dynamic system and then sending out a signal called the emulator response. The comparator sends out an emulator error signal to indicate the difference between the system response and the emulator response. Through a process called backpropagation ${ }^{1}$ the emulator uses this error signal to adjust its response to better match that of the dynamic system. In time, the emulator becomes capabie of responding to control signals just as the dynamic system does.

At this stage, the neural emulator is ready to convert response errors into control errors as desired. The controller is fed a desired response signal and issues the control signal to the dynamic system. The control signal is monitored by the emulator which responds just as the dynamic system would. The emulator response is compared with the desired response to get the response error (see Figure 3). The method of backprogagation is then used to pass the error backward through the neural emulator to get the control error. The control error is then passed backward (again, using backpropagation) through the neural controller to correct its internal weights and consequentiy its control actions. This correctional process is repeated for each of a large number of desired response signals until the neural controller is capable of driving the dynamic system to give the desired response. 


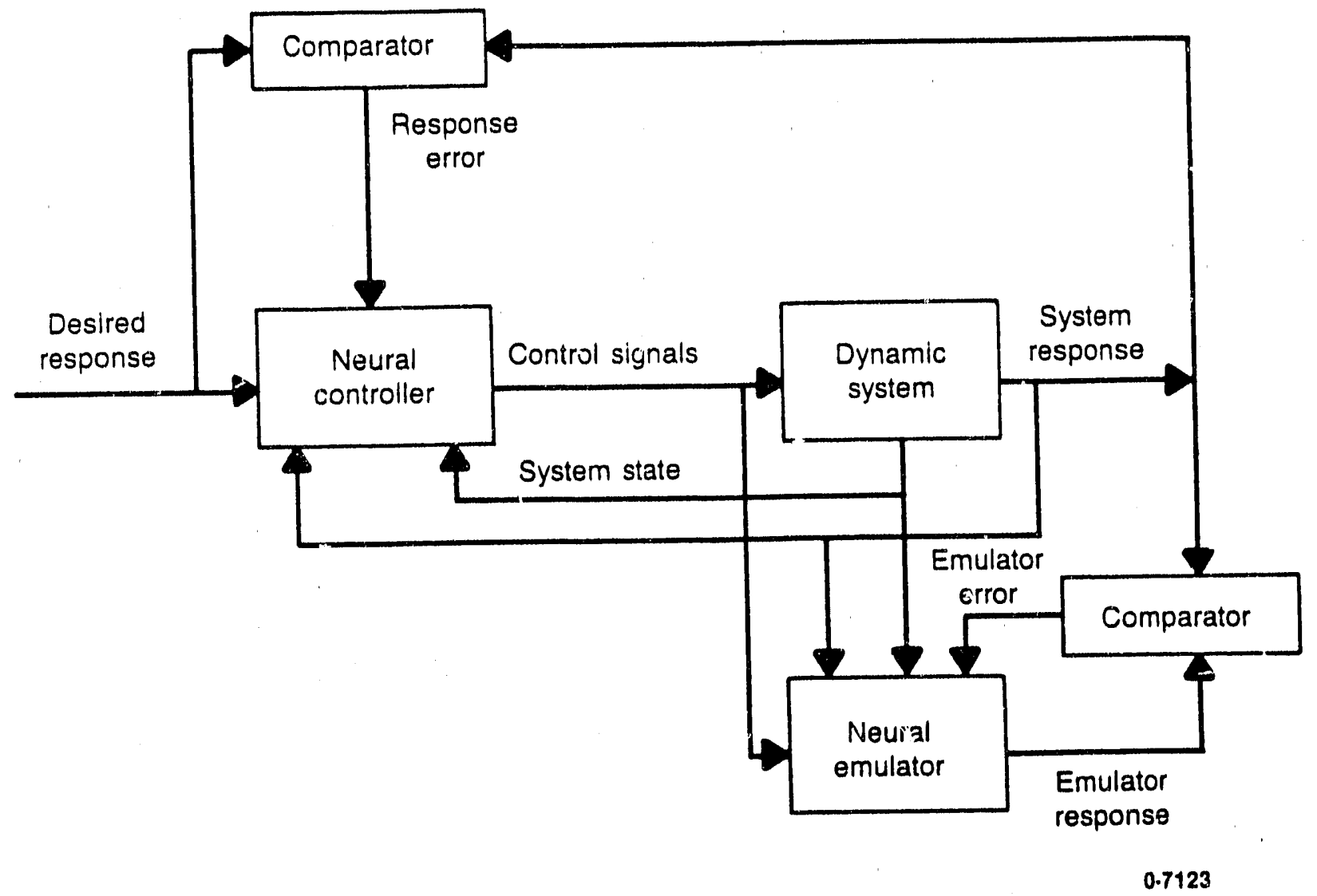

Figure 2. Training the emulator. 


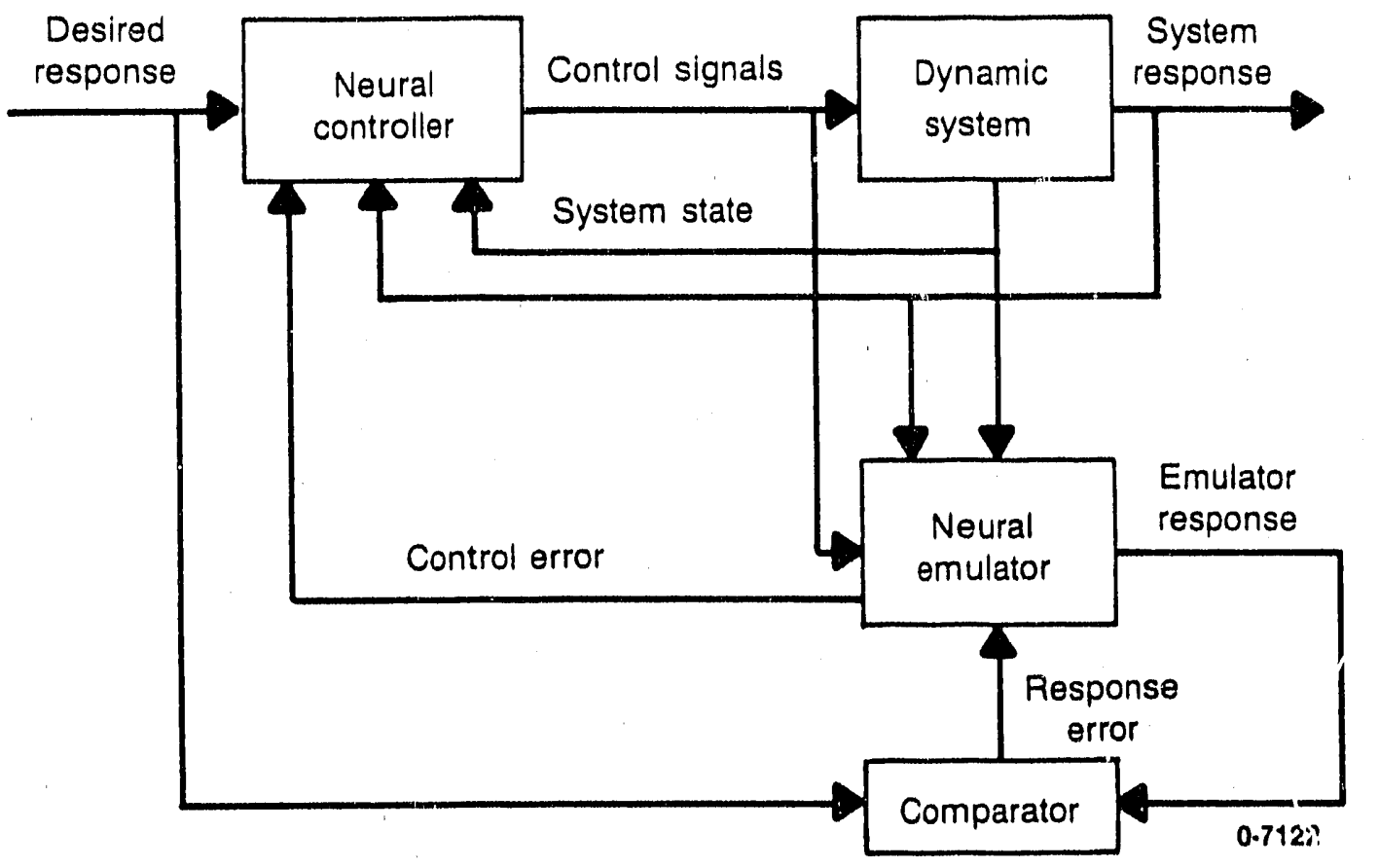

Figure 3. Training the controller. 
For the particular system we are studying, the dynamic system is a couple of interconnected containers or tanks each containing a liquid mixture (such as a salt solution or a mixture of water and antifreeze). The concentration of the mixture in each tank is to be regulated by the neural controller. The controller is capable of turning each tank's inlet valve for this purpose. The process starts when the user specifies the level of concentration for each tank. These specified levels are the desired response shown in the aforementioned diagrams. The concentration level that the controller actually achieves in each tank is called the system response and the inlet valve positions determined by the neural controller are the control signals. The difference between the user-specified concentration levels and the levels achieved by the controller is labeled the controller error in the diagrams.

The valve positions that the controller sends to the dynamic system are al so sent to the neural emulator. The emulator monitors the concentration levels that are currently in the tanks and tries to predict the concentration levels that will result from the valve positions sent by the controller. The prediction made by the emulator is compared with the concentration levels that occur in the tanks at the prediction time to determine the error in the prediction. In the figures, the emulator's prediction is called the emulator response and the error in prediction is called the emulator error.

The following sections give detailed descriptions of the individual components of the control system.

\section{THE DYNAMIC SYSTEM: COUPLED TANKS}

The system of two interconnected tanks is illustrated in Figure 4 . The two tanks shown have two different concentrations of the same solution and are interconnected as shown. Tank 1 is filled by two input flows of fixed but different concentrations. One input is of fixed flow, $f_{d}$, and the other input with flow $f_{a}$ is controlled by $c_{1}$ which is input 1 to the 


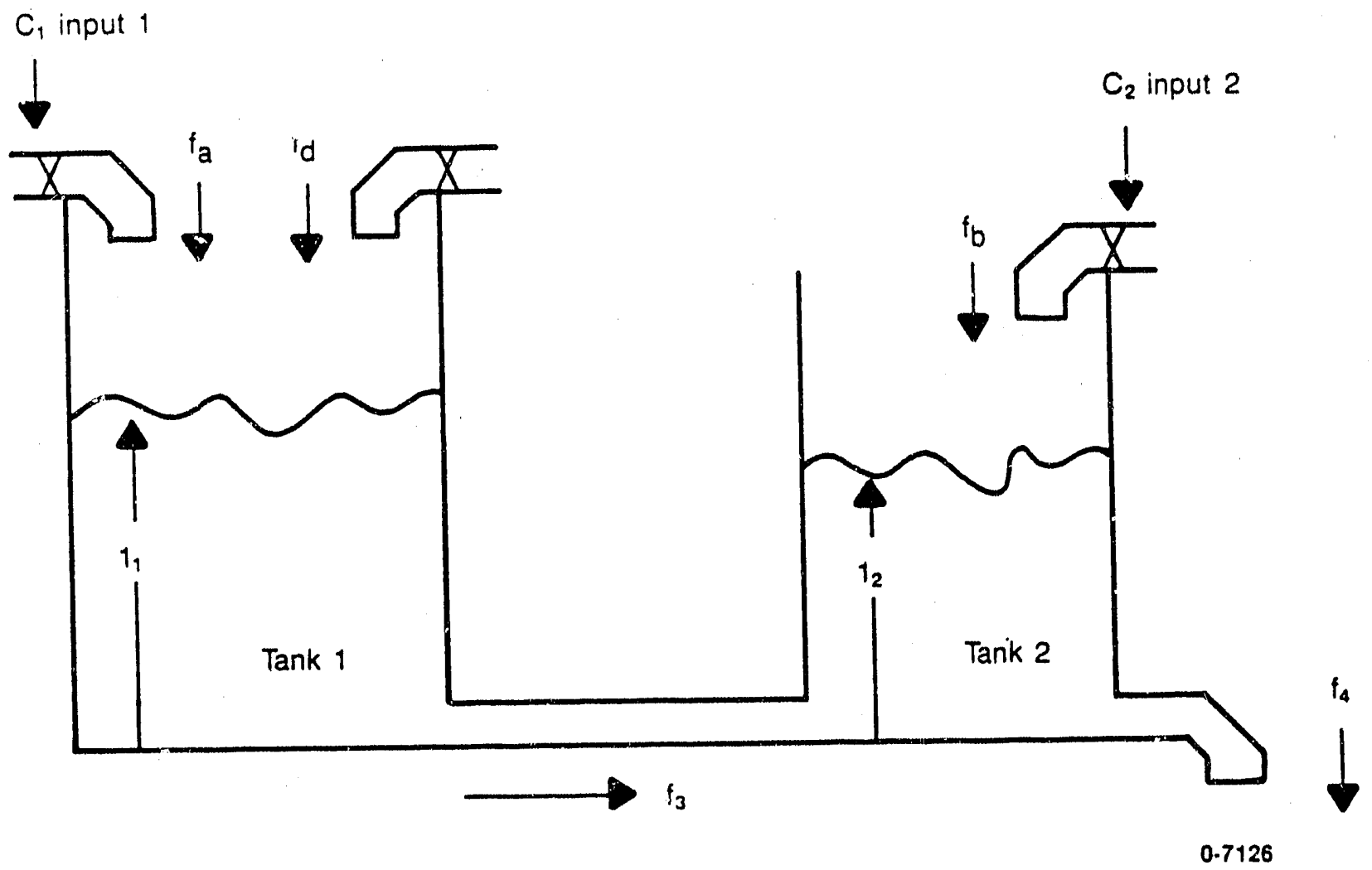

Figure 4. Two-tank System. 
system. The input to tank 2 has fixed concentration and a flowrate which is controlled by $C_{2}$, input 2 to the system. Mixing time in each tank is assumed to be negligible. It is also assumed that operation is such that the tanks do not overflow or go dry during our operation. Of course, eventually, additional control would be needed to insure that assumption.

The dynamics of this system are described by the following differential equations which are developed in Appendix A.

$$
\begin{aligned}
& d l_{1} / d t=b_{10} c_{1}+b_{11} l_{1}+b_{12} l_{2}+b_{00} \\
& d l_{2} / d t=b_{20} c_{2}+b_{21} l_{1}+b_{22} l_{2} \\
& d a_{1} / d t=g_{10} c_{1}+g_{11} Q_{1}+g_{12} a_{1} l_{2} / l_{1}+g_{00} \\
& d a_{2} / d t=g_{20} c_{2}+g_{21} Q_{1}-g_{12} Q_{1} l_{2} / l_{1}+g_{22} a_{2} \\
& s_{1}=h_{1} a_{1} / l_{1} \\
& s_{2}=h_{2} a_{2} / l_{2}
\end{aligned}
$$

where

$I_{1}$ is the liquid height in tank 1

$1_{2}$ is the liquid height in tank 2

$Q_{1}$ is the volume of concentrate in tank 1

$Q_{2}$ is the volume of concentrate in tank 2 .

$C_{1}$ is system input 1

$C_{2}$ is system input 2

$S_{1}$ is the liquid concentration in tank 1 and is system output 1

$S_{2}$ is the liquid concentration in tank 2 and is system output. 2 
The coefficients $b_{00}, b_{10}, b_{11}, \ldots \ldots, b_{22}, g_{10}, g_{11}, \ldots$, , $g_{22}, h_{1}$, and $h_{2}$ are functions of the flows and the tank geometry and are derived in Appendix $A$.

For this system the valves are assumed to have negligible response time and are scaled such that the valve positions, $P_{i}$, are equal to the inputs, $s_{i}$.

\section{COARSE ARCHITECTURE OF THE EMULATOR AND CONTROLLER}

The design of the emulator and controller center around the signals they exchange with the tank system and each other. In what follows, we will show how these signals are represented and how the neural networks that make up the emulator and the controller are constructed. The signals fall into a few different types and are listed here according to category:

1. System variables: The concentration levels that are
a. User-specified $\rightarrow->$ desired response (D).
b. Actually present in tan!:s $\rightarrow->$ system response (S).
c. Predicted by emulator $-->$ emulator response (E).

2. Control variables: Changes in valve-positions that are specified by the controller -.> control signal (C).

3. Valve position variables: Current valve position for each tank valve $\rightarrow>$ valve position $(P)$.

4. Internal system variables: Any internal quantity concerning the tank system that has an effect on the concentration levels. - -> internal system variable (I). 
The letters in parenthes is will be used to denote each signal when it occurs at the input or output of a neural network. The networks will be shown "top-down" fashion. That is, the major portions of the networks will be shown first and then each portion will be broken into subsections. The subsections will then be viewed in detail, etc.

Both the emulator and controller are dual structures as shown in Figure 5. The boxes connected by arrows are groups of "neurons" or "cells". Odd numbered groups are ised to process information concerning tank 1 and even numbered groups are used to process information concernirig tank 2. The arrows indicate the primary direction of information flow. The lowermost boxes are input groups chat receive sigials from the outside and the uppermost boxes are output groups that send signals back. The box in the center (of either the emulator or the controller) is a group of cells that merges and processes the signals from the input groups. These signals are then sent to the outp'st groups.

The controller receives the actual concentration level and the desired concentration level for tank 1 at group 1 . It also receives tank $1^{\prime}$ 's inlet valve position, the concentration level predicted for tank 1 by the emulator. The same tank 2 information is received at group 2. Both group 1 and group 2 receive system variable information for the tank system. The control signals for tank 1 are sent from group 3 and those for tank 2 come from group 4.

In the emulator, group 1 reseives the tank 1 concentration level, the value position for the inlet to tank 1 and the control signal sent to tank 1 from the neural controller. Group 1 also receives predictions about tank 1 made earlier by the emulator. Similarly, group 2 receives the same information about tank 2. Both group 1 and group 2 receive system variable information for the tank system. At the output of the emulator, group 3 sends out the concentration level that is predicted for tank 1 and group 4 does the same for tank 2 . 


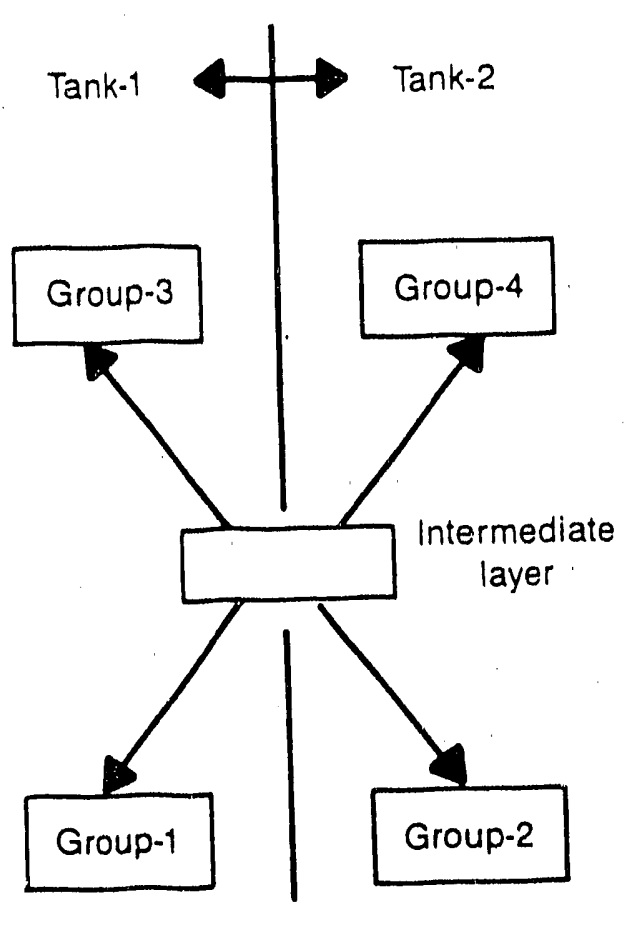

Emulator

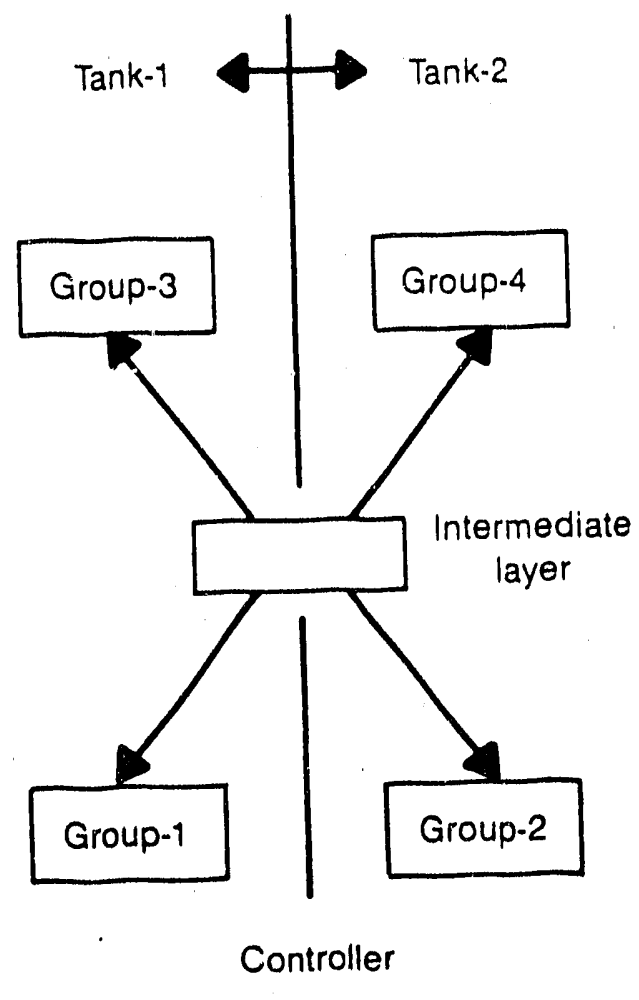

0.7125

Figure 5. Groups of cells in the emulator and controller. 
Since the even numbered groups are structured the same way as the odd numbered groups, the emulator and the controller can each be understood in terms of the odd numbered groups only. Therefore from this point on, only the details of the odd groups will be related. We will consider the emulator input group first. This is group 1 as shown in Figure 6.a. The group is seen as a collection of signals labeled according to the convention above. For instance, a control signal is labeled with the letter "C". In addition however, each signal is broken down into components. This is because each signal contains information about the signal's vaiues over time. For instance, the components of the control signal are labeled C-2, $C-1, C .0, C .1$ and represent the value respectively of the control signal two sample intervals ago, the value one sample interval ago, the present value (the last value sent to the dynamic system and upon which the dynamic system has acted), and the next value (as determined by the controller). Likewise, P-2, P-1, P.0, P.1 are the valve-positions, S-2 through S.0 are the system responses ( $S .1$ will be the dynamic system's response to $C .1$ and so hasn't occurred yet), I-2 through I.0 are the internal parameter values of the system, and E-2 through E.O are the emulator predictions for S-2, S-1, S.0 respectively.

Group 3 (Figure 6.b) of the emulator consists of the emulator output E.1. This is the emulator's prediction of what the system state will be after the dynamic system receives the control signal C.l. It also contains a set of components that look exactly like the components of group 1. During the time that the emulator is learning, it must reproduce a copy in this set of the signals occurring in group 1 . This is just a means of training the group of cells that connect the input groups to the output groups. This set of components has no functional role once the emulator is trained.

For the controller, group 1 is shown in Figure 7.a. This group is similar to that of the emulator with minor differences. The first is the presence of the desired response, $D$. This signal does not change from sample interval to sample interval as do the other parameters so it does not 
Signal components (1-2, 1-1, 1.0 not shown)

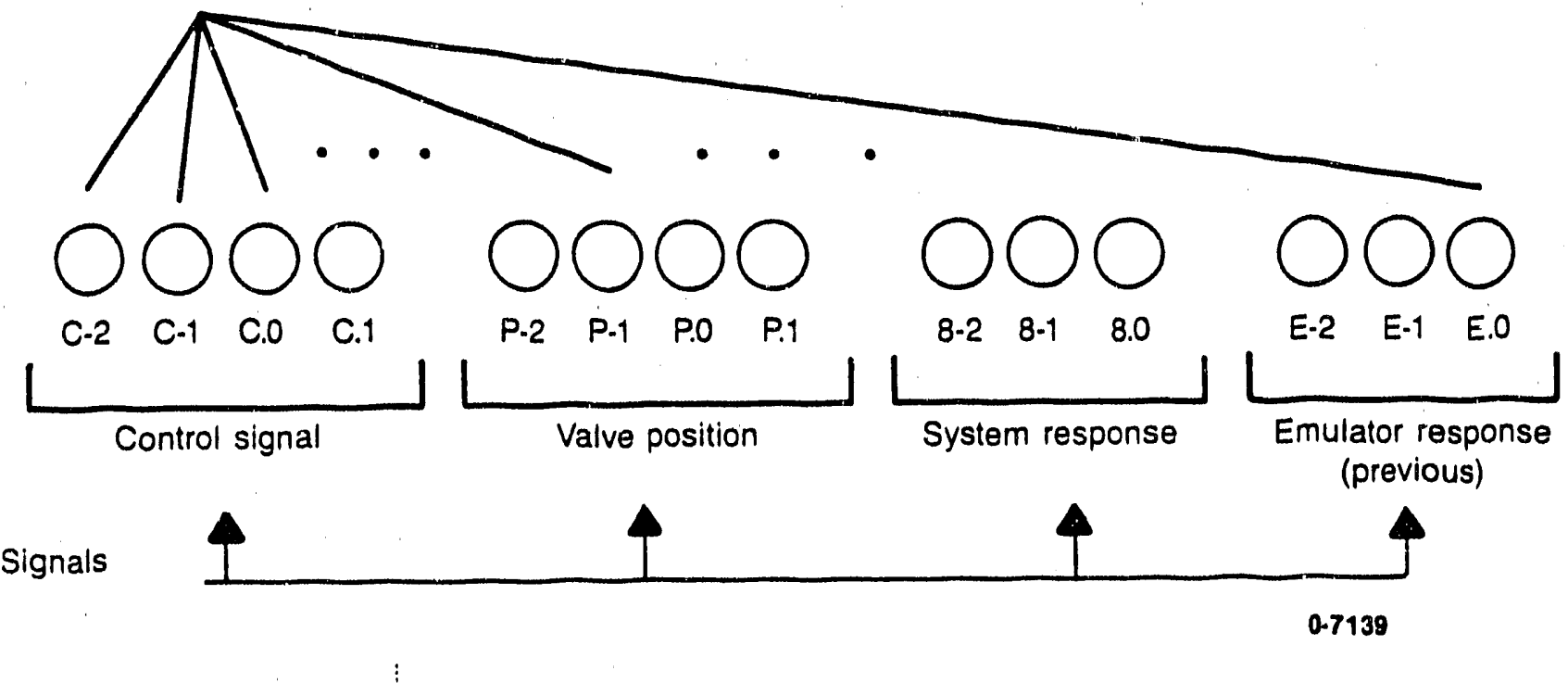

Figure 6a. Emulator input: group-1. 
Components identical to

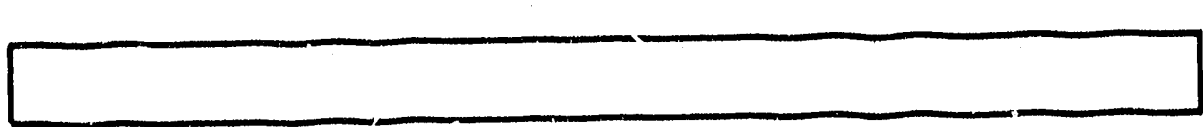

Those of group-1 (training purposes only)

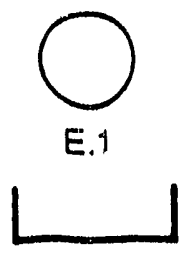

Emulator response (current)

0.7129

Figure 6b. Emulator output: group-3. 
Signal components $(1-2,1-1,1.0$ not shown)

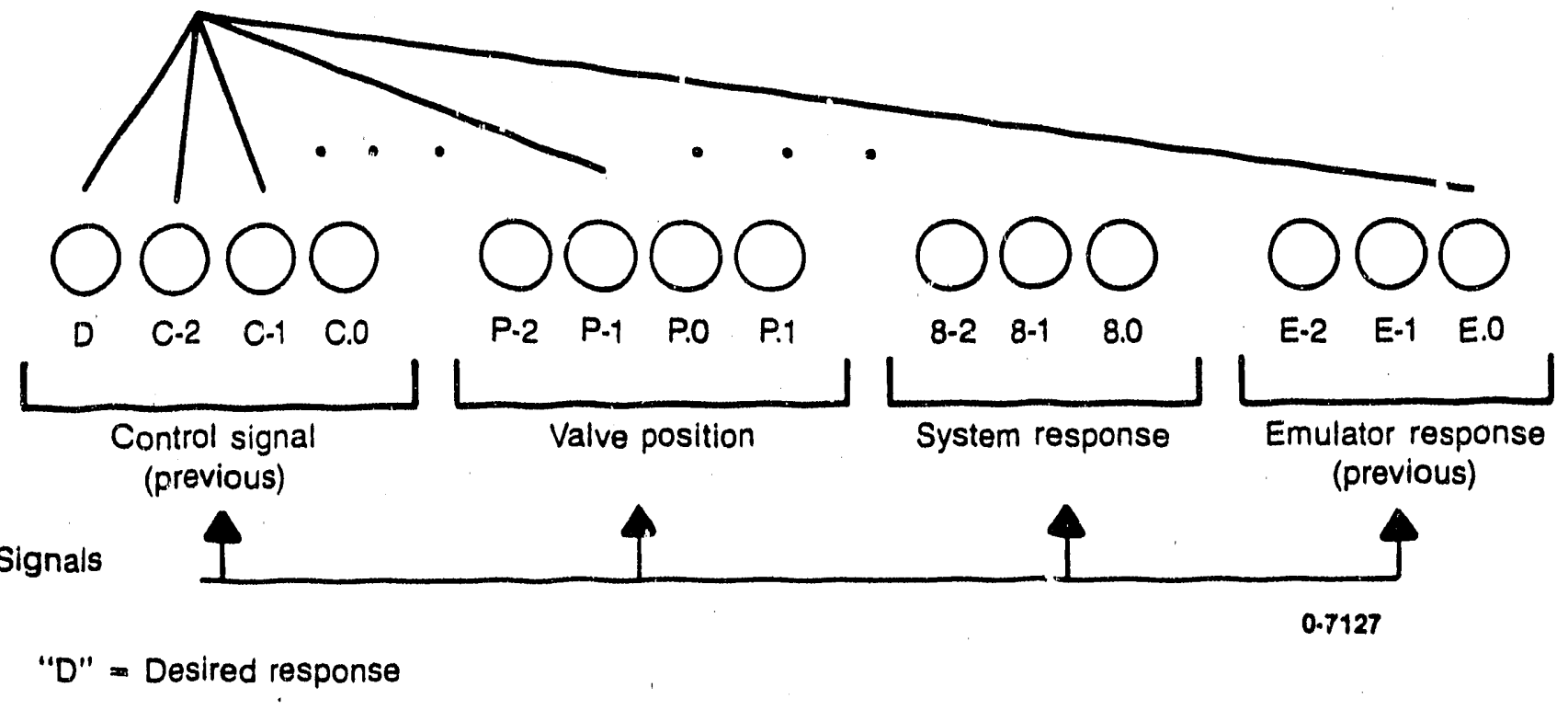

Figure 7a. Controller input: group-1. 
have a numeric subscript. The other difference in the controller input is that C.1 and P.1 are missing since they are determined by th' controller as a function of the group 1 signals shown. Group 3 of the controller is shown in Figure 7.b. In addition to the latest control signal C.1, group 3 contains components identical to those in group 1 of the controller. These are used for training similarly to the use of their counterparts in group 3 of the emulator and have no functional significance once the controller has learned. The parameter P.1 is not an output of the controller since it is determined by adding $C .1$ to the present position P.O of the inlet valve

This level of description of the neural controller and the neural emulator is called the coarse arihitecture since the details of the components (e.g. S-2, C.O, etc.) are not shown. Each of these components is composed of a set of neural cells and the structure of the components in terms of neural cells is called the fine architecture. Knowledge of the coarse architecture is all that is necessary to understanding the principles of the contral system's operation. Therefore, we will discuss the system's operation before we deal with the find architecture.

\section{CONTROL SYSTEM OPERATION}

The control system is started with the dynamic system in some initial state. This initial state specifies the components 5.0 and 1.0 which are respectivaly the concentration level and the internal system variable at the time of start-up. These parameters are assumiad to have had a steady value for all recent sample intervals so that the components S-2 and S-1 are assumed to have the same value as S.0. Likewise the values of I-2 and I-I are the same as those of I.0. The control signals C-2, C-1, C-O start out at zero as do E-2, E-1, and E.0. The desired response, $D$, is set by the user and the controller is then allowed to generate C.1. This is fed to the dynamic system and the emulator which generate the system response S.1 and the emulator response E.1 respectively. 
Components identical to

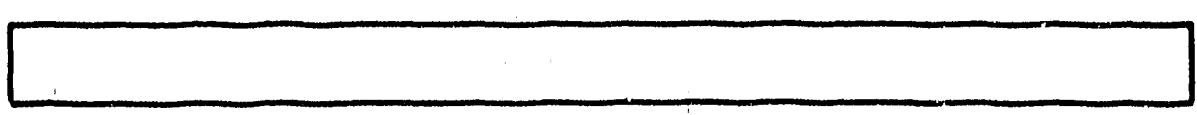

Those of group-1 (training purposes only)

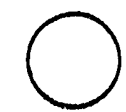

C.1

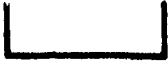

Control

signal

(current)

0.7121

Figure 7b. Controller ouput: group-3. 
What happens next depends upon whether the emulator and controller are learning or have already been trained. In the case that they are being trained, an extra step is involved. Specificaliy, the emulator response E.1 is compared with the system response S.1 to get the emulator error. This error signal is propagated back through the emulator using the method of backpropagation to correct the emulator's response. The correction is effectiveiy stored in the emulator's meniory or weights so that it will make better response in the future (a perfect response is orie exactiy equal to the system response).

In the case that the emulator and controller are not being trained the learning step just mentioned is skipped. In any event, the next step is to update the inputs to the controller and emulator. For this the signals must be shifted. For instance, component $S-1$ is cupied into S-2, S.O is then copied into S-1 and S.1 is copied into S.O. This is done since we are now setting up the control system for the next sample interval; the system response S-1 that had occurred one sample interval ago will in the next instant, be the system response S-2 that occurred two sample intervals ago. This shifting is done for all time subscripted signals, S, I, P, C, E. Figure 8 shows the shifting process. After the inputs are shifted, the controller is allowed to generate another control signal, that is a new value for $C .1$ and the whole process, called a cycle, repeats.

If the controller is being trained during this process, a means of correcting its actions must be provided. For each cycle, if the emulator error is not too large, the emulator response (or perhaps the system response, since low emulator error means that the two are nearly the same) is subtracted from the desired response to get a response error. This error is then back propagated through the emulator without making changes to the emulator's internal parameters to get an error signal at the output of the controller. This is called the control error and its production during controller training is the sole purpose of the emulator. This error signal 

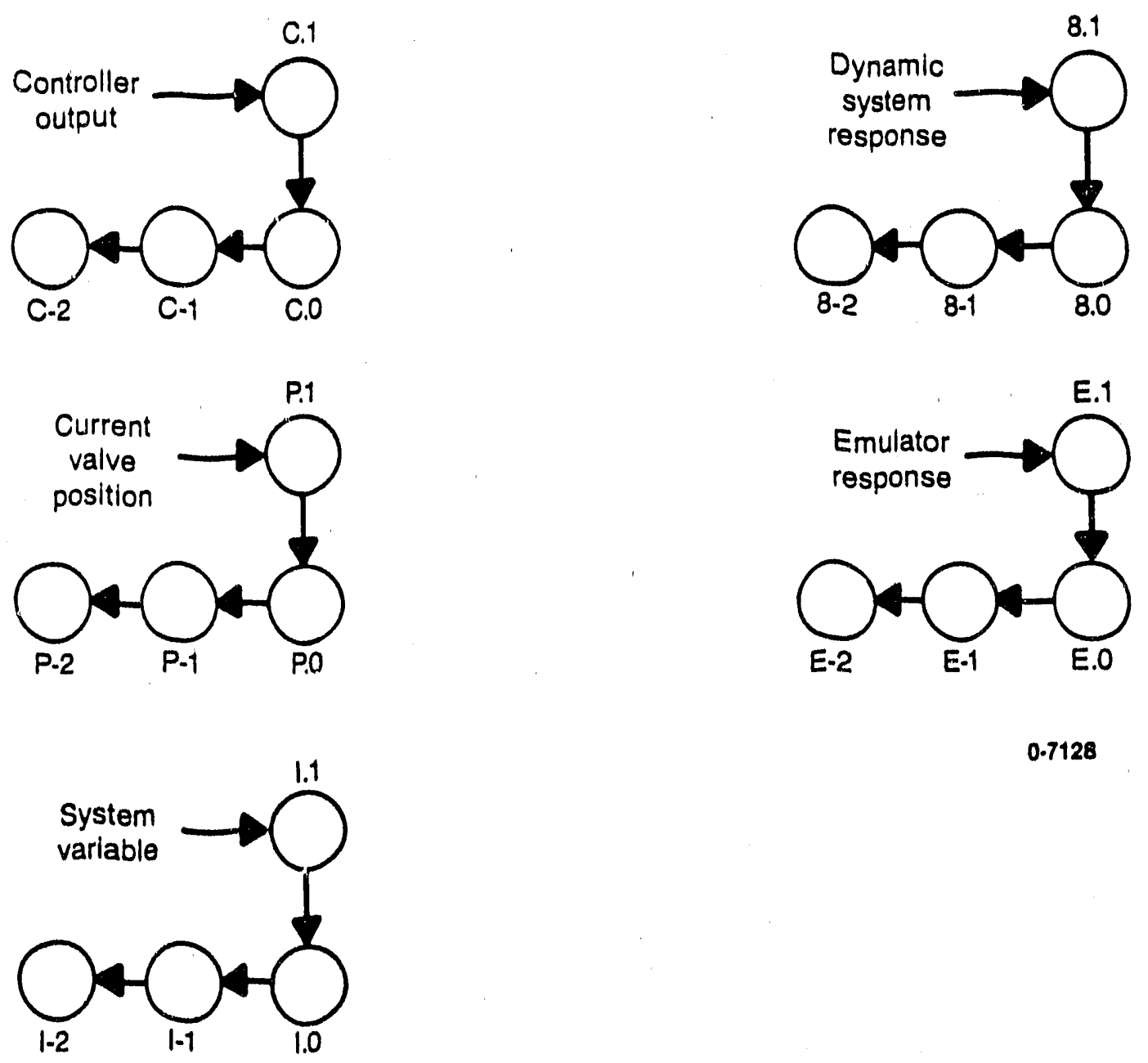

Figure 8. Shifting signal components. 
is back propagated through the controller to change its internal parameters or weights so that it will correct its control strategy. The per-cycle generation of controller corrections is called short-term correction.

After some predetermined number of cycles called an epoch, either the controller has succeeded in putting the dynamic system into a steady state (non-changing condition) or it is still "fumbling at the controls". In the first case, the instant a steady state is reached (i.e. $S-2=s-1=S .0$ ), the system response is subtracted from the desired response to get a response error signal. This is back propagated through the emulator as usual to get the control error signal and the latter is back propagated through the controller to correct its strategy. In the case that the controller is still fumbling at the controls it is more difficult to determine an appropriate error signal for correction. For the present, the system is stopped and a new initial state and desired response is given to the system. The old initial state and desired response is given to the system at a letter time after it has had more training. In any event, the training that occurs after each epoch in this way is called long-term correction.

Some details are to be noted about controller training. First, the control signal is to be scaled-up if the emulator error is small and scaled-down if the emulator error is large. This keeps the controller from adjusting its internal parameters when the emulator is in its early stages of learning and cannot provide the controller with a reliable control error signal. Also, the short-term training of the controller will have the property of "punishing" the controller when an action it makes takes the dynamic system farther from the desired response than it was previously. This should increase the tendency of the controller to get the dynamic system into a steady state by the end of an epoch as desired. By monitoring the history of the system response and its movement toward/away from the desired response, the desired effect could amplified by scaling up the 
control error signal when the controller starts moving the dynamic system away from the desired response. The result should be a controller that quickly learns to bring about the desired response from the dynamic system.

\section{FINE ARCHITECTURE OF THE EMULATOR AND CONTROLLER}

All components of the emulator and controller are made up of neural cells or simply units. The fine architecture is the specification of the way in which the units compose the units. Figure 9 shows this structure for each signal S, I, P, C, E. In the figure, each unit has a weighted value or value shown beneath it. Also each unit has an activation level between zero and ore (see Reference 1, Ch. 8). U: like the unit's value, the activation changes over time as the signals in the control system change.

For our purposes, a unit is considered to be "on" when it has an activation-level above 0.5 and is "off" otherwise. For a system response, or "S" component (e.g. S.0), there are 5 units as shown. The value of S.O is given by the sum of the values of its on units. For example, if the third and fifth units of S.0 are on and the others off then the value of 5.0 is $1 / 4+1 / 16=5 / 16$. Of course, $S .0$ is an input node, so if we know that its value, rounded to the nearest $1 / 16$, is $5 / 16$, then the third and fifth units are turned on to indicate this value. For the other component types, the encoding of values is similar though the number of units vary from type to type as do their associated values. In particular, the control components can assume negative as well as positive values. Therefore, a unit is added that indicates the sign of the component. For all component types, the method of encoding was chosen to span the possible range of values of the component and to yield the precision necessary to accurately represent the component's value. As can be seen, the " $C$ " components require the most precision and the " $i$ " components have the widest range of possible values. 

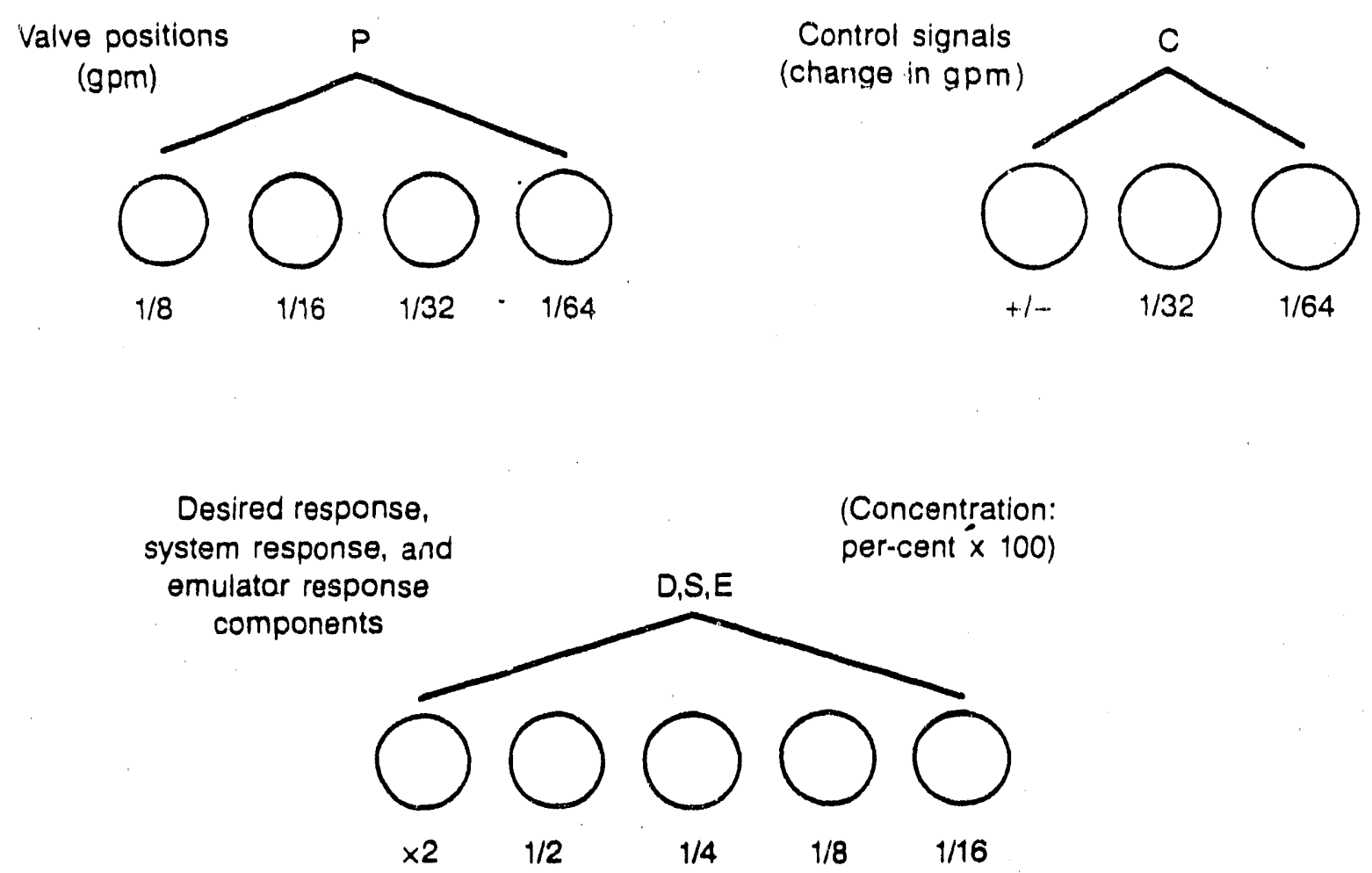

System variables

(rate-change of concentration:

per-cent $\times 100$ per min.)

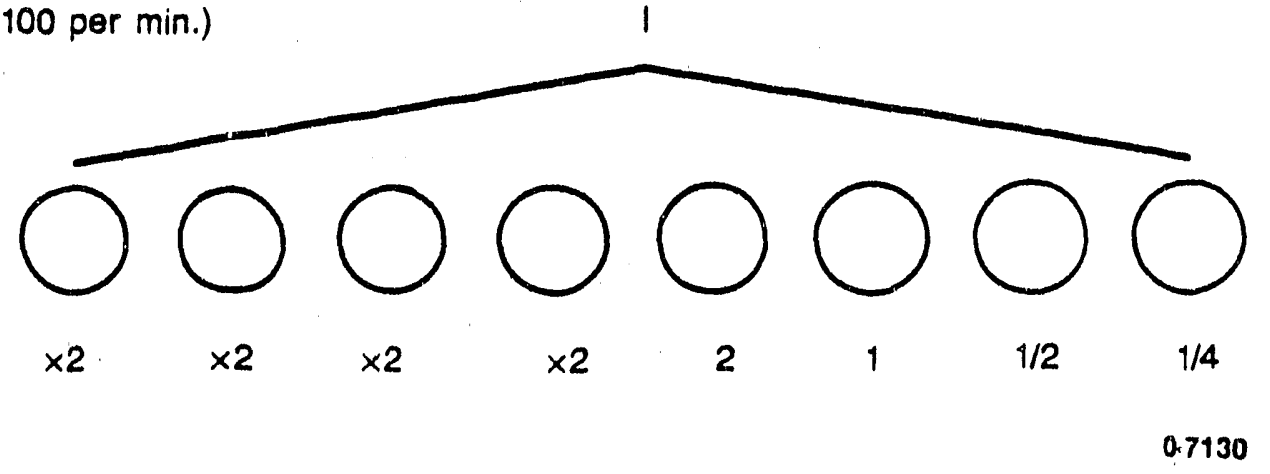

Figure 9. Structure of signal components

( $x$ 2 units multiply value of other units by 2 when on). 
The units that make up signal components at the input of the emulator or controller are called input units and those that make up signal components at the output of the emulator or controller are called the output units. The encoding scheme mentioned in the previous paragraph dictates how the input units are to be assigned activation levels as a function of the value of the signal component they represent and it prescribes how the output unit activations that occur are to be interpreted as values for the component represented by the units. By keeping track of signal component values and converting them into input unit activation levels, signal information can be passed to the emulator or controller from outside. Conversely, converting output unit activations into signal component values, signal inforriation can be passed from the emulator or controller to other parts of the control system.

Knowledge of the fine architecture of the emulator and controller completes the design of each of these neural networks. The only thing left to relate is the fact that the middle group of units that tie the input units to the output units is merely a set of 10 to 20 units. The actual number of units is at present a variable to be optimized during experimentation with the control system. The units are connected in layers as done in standard backpropagation networks.

Assuming that 15 units are used in the middle layer of each network, the total number of units composing the emulator and controller can be calculated. In the case of the emulator, the group 1 units number 82 . We add 5 more to get 87 for the number of units in group 3 . The even numbered groups have the same number of units as their odd counterparts so the total number of units in the emulator is 353 . For the controller, the total comes to 341 .

This unit count is important for understanding the amount of computer time required to train a controller to reliably regulate the dynamic system. Entire epochs must be simulated for each desired response signal to 
be trained in combination with each initial state of the dynamic system to be trained. This implies that although pilot simulations can be done on mini and mainframe computers, the system will ultimately have to be simulated on high speed supercomputers in order to develop the principles of design, optimize the architecture and validate this control method.

\section{SUMMARY}

Neural networks have been investigated for use as a trainable controller for feedback control systems. A methodology has been developed that designs the controller by first emulating the dynamic system to be controlled. A technique called backpropagation is used to design the emulator then is also used along with the emulator to design the controller itself. Two advantages that a neural network controller has over a conventional controller are that it can be retrained as the system it is controlling changes and because a neural network is a nonlinear system it may give better results for nonlinear dynamic systems. Further work is needed to determine the extent of the advantages when the nonlinear system is controlled. 


\section{REFERENCES}

1. D. E. Rumelhart, J. L. McCle1land, and the PDP Research Group, Parallel Distributed Processing: Explorations in the Microstructure of Cognition, Vo1. 1: Folindations, MIT Press, Cambridge, Massachusetts, 1986. 


\section{A.PPENDIX A}

T'NO TANK SYSTEM DYNAMICS 


\section{APPENDIX A}

\section{TWO TANK SYSTEM DYNAMICS}

Figure Al shows a system of two iriterconnected tanks. The same liquid is in each tank except for the concentration of the soiution. The concentration in each tank is separately controlled by the input flow rates. Because input flows can not be negative there are constraints on the tank concentrations and flows. For this example, it is assumed that the operation is such that the tanks are large enough that they will not overflow and are operated with initial liquid levels so that the tanks do not go dry.

The input flows to tank 1 are $f_{a}$, which is controlled by system input signal 1 , and $f_{d}$, which is a fixed flow rate. These flows have fixed but different concentrations. The output flow from tank 1 is $f_{3}$. which is also an input to tank 2. This causes coupling of the dynamics of the two tanks. The second input flow to tank 2 is $f_{b}$, which is controlled by system input 2 . The output flow of tank 2 is $f_{4}$.

The dynamics of the tank system are gizen by the liquid levels and the volume of concentrate in each tank. The differential equations describing these dynamics are developed below.

The liquid levels are described by

$$
\begin{aligned}
& d l_{1} / d t=\left(f_{a}+f_{d}-f_{3}\right) / a_{1} \\
& d l_{2} / d t=\left(f_{b}+f_{3}-f_{4}\right) / a_{2}
\end{aligned}
$$

where

$1_{1}=$ the liquid level in tank 1

$1_{2}=$ the liquid level in tank 2

$f_{a}$ : the flow rate of the controlled flow to tar. 1

$f_{b}=$ the flow rate of the controlled flow to tank 2 


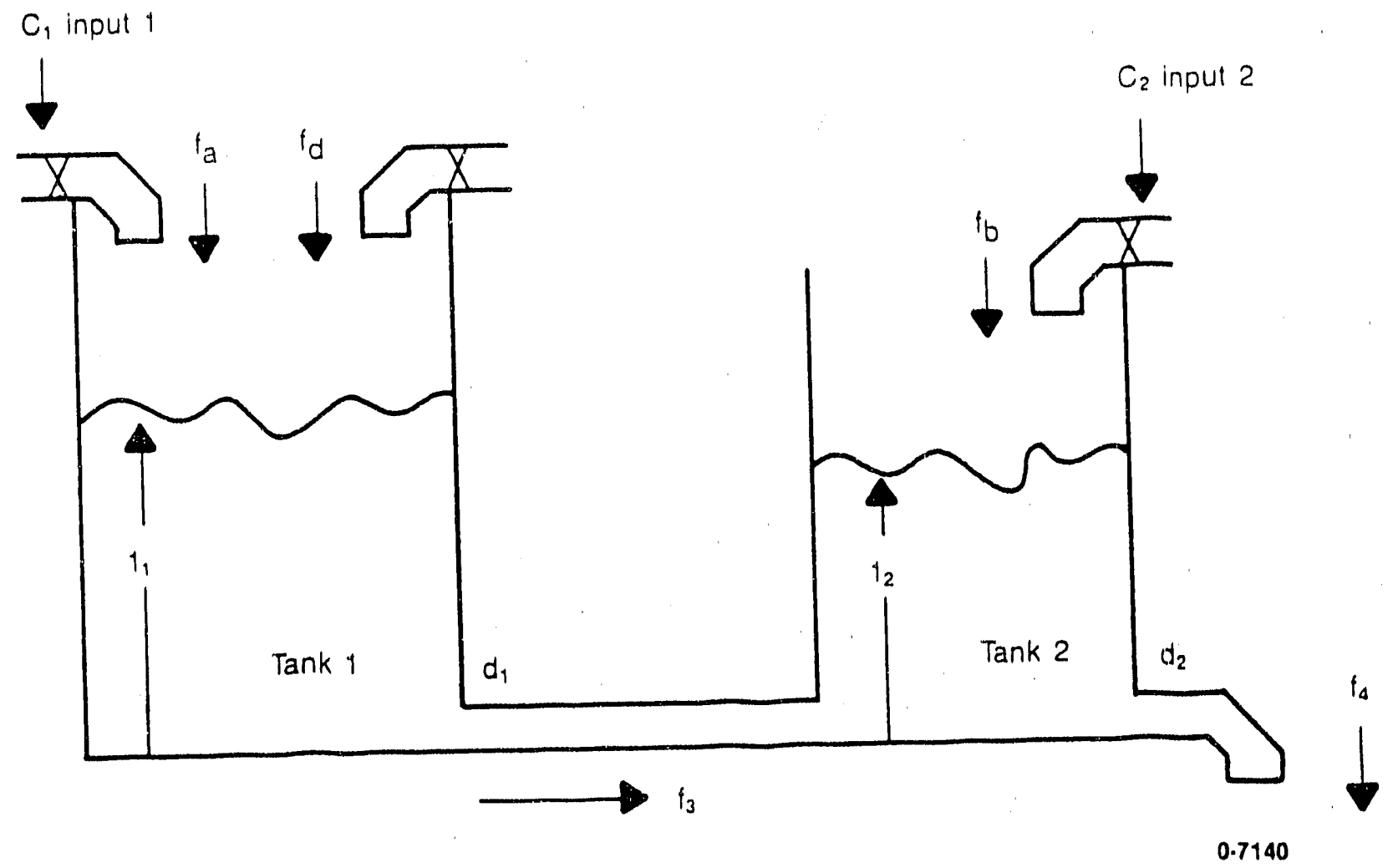

Figure A 1 Two-tank system. 
$f_{d}=$ the flow rate of the fixed flow to tank 1

$f_{3}=$ the flow rate of the output flow of tank 1

$f_{4}=$ the flow rate of the output flow of tank 2

$a_{1}=$ the cross sectional area of tank 1

$a_{2}=$ the cross sectional area of tank 2

We can express $f_{3}$ and $f_{4}$ as functions of the liquid levels by the following equations:

$$
\begin{aligned}
& f_{3}=\left(1_{1}-1_{2}\right) / d_{1} \\
& f_{4}=1_{2} / d_{2}
\end{aligned}
$$

where $d_{1}$ and $d_{2}$ are the flow resistances of the output openings of tanks 1 and 2. Since the input flows $f_{a}$ and $f_{b}$ are controlled by the system input signals, they may be expressed as:

$f_{a}=b_{10} a_{1} c_{1}$

$f_{b}=b_{20} a_{2} c_{2}$

where

$C_{1}=$ system input signal 1

$c_{2}=$ system input signal 2

$b_{10}=$ scaling factor between $f_{a}$ and $c_{1}$

$b_{20}=$ scaling factor between $f_{b}$ and $c_{2}$

Substituting Equations (3) and (5) into Equation (1) and Equations

and (6) into Equation (2) gives:

$$
\begin{aligned}
& d 1_{1} / d t=b_{10} c_{1}+b_{11} l_{1}+b_{12} l_{2}+b_{00} \\
& d 1_{2} / d t=b_{20} c_{2}+b_{21} 1_{1}+b_{22} l_{2}
\end{aligned}
$$


where $b_{10}$ and $b_{20}$ are given above and

$$
\begin{aligned}
& b_{00}=f_{d} / a_{1} \\
& b_{11}=-1 /\left(a_{1} d_{1}\right) \\
& b_{12}=1 /\left(a_{1} d_{1}\right) \\
& b_{21}=1 /\left(a_{2} d_{1}\right) \\
& b_{22}=-1 /\left(a_{2} d_{1}\right)-1 /\left(a_{2} d_{2}\right)
\end{aligned}
$$

The quantity of concentrate in each tank is given by

$$
\begin{aligned}
& d Q_{1} / d t=p_{a}+p_{d}-p_{3} \\
& d Q_{2} / d t=p_{b}+p_{3}-p_{4}
\end{aligned}
$$

where

$Q_{1}=$ the volume of concentrate in tank 1

$Q_{2}=$ the volume of concentrate in tank 2

$p_{a}=$ the rate at which the concentrate is entering tank 1 via $f_{a}$ $P_{b}=$ the rate at which the concentrate is entering tank 2 via $f_{b}$ $p_{d}=$ the rate at which the concentrate is entering tank 1 via $f_{d}$ $p_{3}=$ the rate at which the concentrate is leaving tank 1 via $f_{3}$ $p_{4}=$ the rate at which the concentrate is leaving tank 2 via $f_{4}$

To write $p_{a}, p_{b}, p_{3}$, and $p_{4}$ in terms of the flow rates note that

$$
\begin{aligned}
& p_{a}=c_{a} f_{a} \\
& p_{b}=c_{b} f_{b} \\
& p_{3}=c_{1} f_{3} \\
& p_{4}=c_{2} f_{4}
\end{aligned}
$$


where

$c_{a}=$ the fractional concentration of $f_{a}$

$c_{b}=$ the fractional concentration of $f_{b}$

$c_{1}=$ the fractional concentration of the liquid in tank 1

$c_{2}=$ the fractional concentration of the liquid in tank 2 .

Note that the concentrations are given by lower case letters and the system input signals by upper case letters so the $c_{1}$ is a concentration and $C_{1}$ is an input signal.

Equations (9) and (10) can now be written as

$$
\begin{aligned}
& d Q_{1} / d t=c_{a} b_{10} a_{1} c_{1}+p_{d}-c_{1}\left(1_{1}-1_{2}\right) / d_{1} \\
& d Q_{2} / d t=c_{b} b_{20} a_{2} c_{2}+c_{1}\left(1_{1}-1_{2}\right) / d_{1}-c_{2} l_{2} / d_{2} \\
& \quad \text { Now note that } c_{1} \text { and } c_{2} \text { are functions of } 1_{1}, 1_{2}, Q_{1} \text {, and } Q_{2} \\
& \text { as shown by } \\
& c_{1}=Q_{1} / v_{1} \\
& c_{2}=Q_{2} / v_{2}
\end{aligned}
$$$$
d Q_{2} / d t=c_{b} b_{20} a_{2} c_{2}+c_{1}\left(1_{1}-1_{2}\right) / d_{1}-c_{2} 1_{2} / d_{2}
$$

where

$V_{1}=$ volume of liquid in tank 1
$V_{2}=$ volume of liquid in tank 2

and

$$
\begin{aligned}
& v_{1}=1_{1} a_{1} \\
& v_{2}=1_{2} a_{2} .
\end{aligned}
$$


Therefore,

$$
\begin{aligned}
& c_{1}=Q_{1} /\left(1_{1} a_{1}\right) \\
& c_{2}=Q_{2} /\left(1_{2} a_{2}\right) .
\end{aligned}
$$

These are also the outputs of the system, therefore,

$$
\begin{aligned}
& s_{1}=c_{1}=h_{1} a_{1} / l_{1} \\
& s_{2}=c_{2}=h_{2} a_{2} / l_{2}
\end{aligned}
$$

where

$S_{1}=$ system output 1

$S_{2}=$ system output 2

$h_{1}=1 / a_{1}$

$h_{2}=1 / a_{2}$.

Making substitutions for $c_{1}$ and $c_{2}$ in Equations (11) and (12) gives

$$
\begin{aligned}
& d Q_{1} / d t=c_{a} b_{10} a_{1} c_{1}+p_{d} \cdot a_{1} /\left(a_{1} d_{1}\right)+a_{1} l_{2} /\left(a_{1} d_{1} l_{1}\right) \\
& d Q_{2} / d t=c_{b} b_{20} a_{2} c_{2}+a_{1} /\left(a_{1} d_{1}\right)-a_{2} /\left(a_{2} d_{2}\right)-a_{1} l_{2} /\left(a_{1} d_{1} l_{1}\right)
\end{aligned}
$$

Combining constants gives

$$
\begin{aligned}
& d Q_{1} / d t=g_{10} C_{1}+g_{11} Q_{1}+g_{12} a_{1} l_{2} / l_{1}+g_{00} \\
& d Q_{2} / d t=g_{20} C_{2}+g_{21} Q_{1}+g_{22} a_{2}-g_{12} Q_{1} l_{2} / r_{1}
\end{aligned}
$$


where

$g_{00}=p_{d}$

$g_{10}=c_{a} b_{10}{ }^{a} 1$

$g_{11}=-1 /\left(a_{1} d_{1}\right)$

$g_{12}=1 /\left(a_{1} d_{1}\right)$

$g_{20}=c_{b} b_{20} a_{2}$

$g_{21}=g_{12}$

$g_{22}=-1 /\left(a_{2} d_{2}\right)$

As can be seen from Equations (23) and (24), this system is non-linear and is difficult to analyze analytically. Because of this, techniques to design standard controllers are not commonly available if at all and a neural network controller offers a real advantage of working with nonlinear systems. 

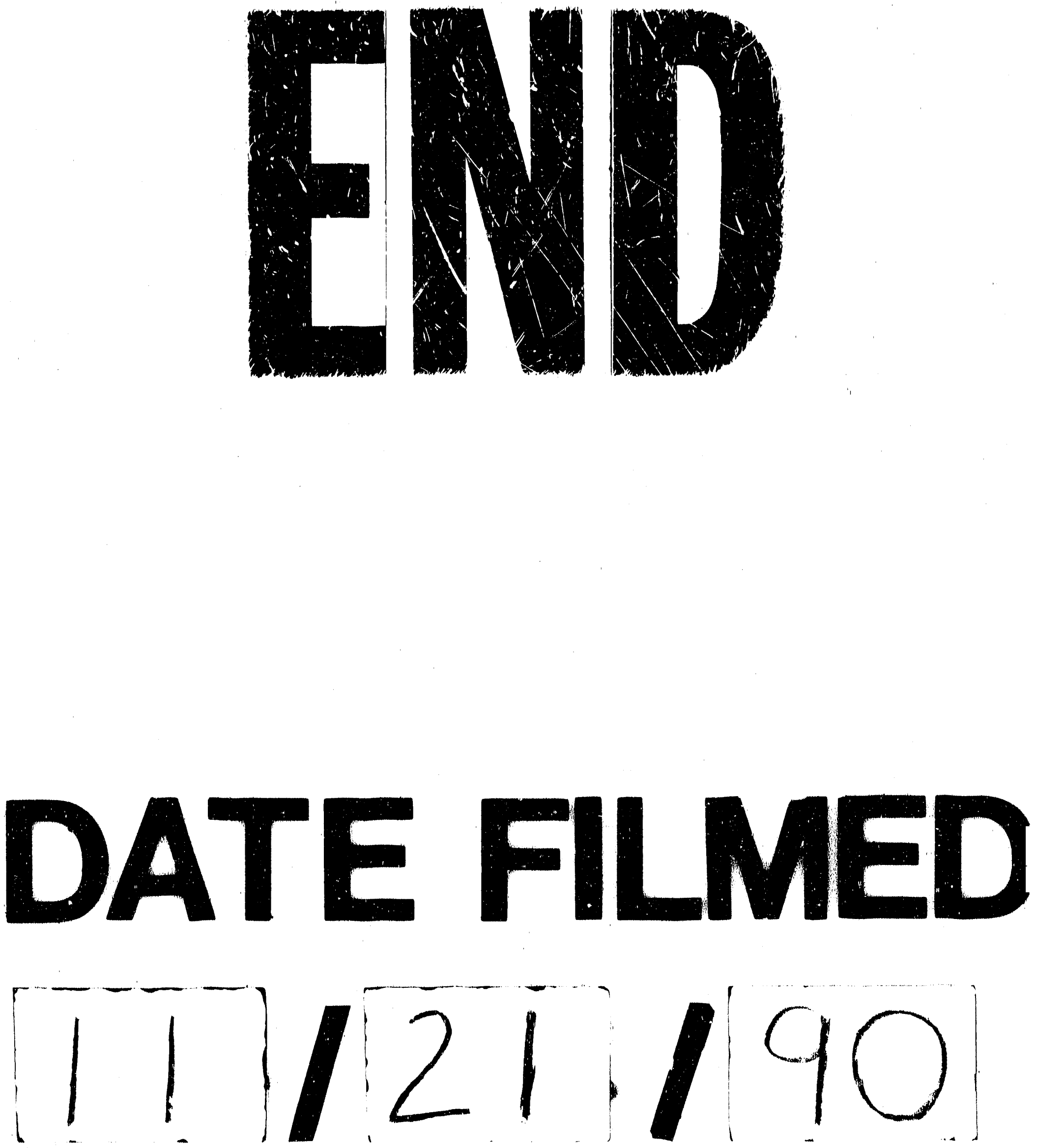